


\title{
Evaluation of Apical Filling after Warm Vertical Compaction using Two Different Endodontic Materials: Resilon ${ }^{\circledR}$ and Gutta-Percha
}

\author{
Carla Zogheib, Alfred Naaman, Etienne Medioni
}

\begin{abstract}
Forty-eight extracted maxillary anterior teeth instrumented with ProTaper system to apical preparations $0.25,0.40$ and 0.60 $\mathrm{mm}(\mathrm{n}=16$ each) were divided into six groups for filling as following: Group 1: Apical preparation 25/100 + gutta-percha/ AH Plus sealer, group 2: Apical preparation 25/100 + Resilon/ Real Seal, group 3: Apical preparation 40/100 + gutta-percha/ AH plus sealer, group 4: Apical preparation 40/100 + Resilon/ Real Seal, group 5: Apical preparation 60/100 + gutta-percha/ AH plus, group 6: Apical preparation 60/100 + Resilon/Real Seal. All teeth were filled using the warm vertical technique. Horizontal sections at 1, 2 and $3 \mathrm{~mm}$ from the apex were observed by SEM under $200 \times$ magnification. Sections were digitally photographed under a stereomicroscope and the images were transferred to a compatible PC for image analysis. The surface area of voids were calculated and compared at all levels; using the Kruskal-Wallis test with Sidak correction and Mann-Whitney $U$ test $(p>0.05)$.

Comparisons within each group (gutta-percha/ $\mathrm{AH}$ plus and Resilon/Real Seal) showed the presence of voids but there was no significant difference between any level of sectioning for apical preparations of 25 and $40(p>0.05)$. The only difference was in the groups prepared to size 60 and filled with Resilon/ Real Seal which showed significantly more voids than the group filled with gutta-percha/AH plus and especially at the $3 \mathrm{~mm}$ level.
\end{abstract}

Conclusion: The system Resilon/Real Seal did not achieve better results in terms of sealing ability in the apical third when compared to the conventional gutta-percha/AH plus sealing system.

Keywords: Apical seal, Gutta-percha, Obturation, Resilon, Sealer, Voids.

How to cite this article: Zogheib C, Naaman A, Medioni E. Evaluation of Apical Filling after Warm Vertical Compaction using Two Different Endodontic Materials: Resilon ${ }^{\circledR}$ and Gutta-Percha. World J Dent 2012;3(2):166-170.

Source of support: Nil

Conflict of interest: None declared

\section{INTRODUCTION}

Complete obturation of the root canal with an inert filling material and creation of a fluid-tight seal are among the major goals of successful endodontic treatment. ${ }^{1}$ Gutta-percha is the obturation material of choice, ${ }^{2}$ it is used with various filling techniques and associated with different types of sealers. Gutta-percha sealers are used to attain an impervious seal between the core material and root canal walls. Ideally, these materials should seal the canal laterally and apically and have good adaptation to root canal dentine. ${ }^{3}$
But the major inconvenient of gutta-percha is that it does not adhere to canal walls. It would, thus, be advantageous to replace conventional root canal fillings made with gutta-percha and sealer by sealers and core materials with adhesive properties.

Recently, Resilon obturation system was introduced. ${ }^{4,5}$ This product is used in combination with a self-etching primer to create a solid monoblock. Resilon is a polymerbased thermoplastic resin material and is used in conjunction with the Epiphany Root Canal Sealant (Pentron Clinical Technologies, Wallingford, CT, USA) or Real Seal (Sybron Endo, Orange, CA, USA) which is a dual-curable resin material containing dimethacrylates. The primer of Resilon is an aqueous solution of an acidic monomer. It can be used with any of the popular gutta-percha root canal obturation techniques: Lateral condensation, vertical compaction, continuous wave and thermoplasticized guttapercha.

Previous studies ${ }^{5,6}$ have evaluated various aspects of this new obturation system, including microbial leakage, bond strength, sealing ability and fracture resistance. However, it might also be hypothesized that a methacrylate-based sealer, such as Real Seal is stable over time when applied properly, but the filling should not contain unfilled areas (voids).

\section{OBJECTIVES}

In light of these observations, after modifying and enlarging the apical diameter to size 25, 40 and $60 / 100 \mathrm{~mm}$, the purpose of this study was to investigate and compare the cross-sectional area of sealer and voids along the apical $3 \mathrm{~mm}$ of the root canal, filled with vertical compacted guttapercha/AH plus sealer and Resilon/Real Seal. The goal will be to determine whether enlarging the apex will result to a better sealing ability and consequently less voids between gutta-percha/AH plus and Resilon/Real Seal.

\section{MATERIALS AND METHODS}

\section{Specimen Preparation and Obturation}

Forty-eight freshly extracted anterosuperior teeth were selected. Access openings were prepared into the pulp chamber, instrumentation was completed with rotary 
ProTaper files (Dentsply Maillefer, Ballaigues, Switzerland) in a crown down technique. Irrigation with $5.25 \%$ sodium hypochlorite and patency filing were carried out between each instrument. At the end of each canal preparation a $5 \mathrm{ml}$ rinse of $17 \%$ EDTA was performed, followed by a final rinse with water.

The roots were divided randomly into six groups of eight each prepared and filled as follows:

- Group 1 (Gutta-percha/AH plus, apical preparation 25/100 mm): The F2 ProTaper was taken to length in eight canals. The diameter of the apical preparation resulting was 25/100 $\mathrm{mm}$. A gutta-percha master cone medium size (Henry Schein, USA) was lightly coated with $\mathrm{AH}$ plus sealer and was inserted $0.5 \mathrm{~mm}$ short of the working length with tug-back. Vertical compaction for the apical $4 \mathrm{~mm}$ was performed with the element (Analytic Technology, SybronEndo Corporation, Orange, Ca, USA) at $200^{\circ} \mathrm{C}$. The middle and cervical portion of the root canals were back-filled with the Obtura II device (Obtura/Spartan, Fenton, MO, USA) preset to $180^{\circ} \mathrm{C}$ and $1 \mathrm{~mm}$ below the cementoenamel junction, each time injecting a 3 to $4 \mathrm{~mm}$ segment and compacting the gutta-percha with prefitted hand pluggers.

- Group 2 (Resilon/Real Seal, apical preparation size 25/ $100 \mathrm{~mm}$ ): Eight root canals prepared to a size 25 were filled with a Medium Resilon cone. The cone was trimmed to give tug-back $0.5 \mathrm{~mm}$ short of the working length. A self-etching primer (Real Seal) was placed in the root canal using a microbrush (Microbrush International, Grafton, WI, USA) and paper points (Roeko, Langenau, Germany) soaked with the primer. After 30 seconds, excess primer was removed with paper points. The Resilon master cone was lightly coated with Real Seal and placed into the root canal. Vertical compaction was then applied with the element at a temperature of $150^{\circ} \mathrm{C}$ filling the apical $4 \mathrm{~mm}$. The middle and cervical part were filled with the Resilon Extruder cartridge (Sybron Endo) at $150^{\circ} \mathrm{C}$ the same way as group 1 .

- Group 3 (Gutta-percha/AH plus, apical preparation size 40/100 mm): F4 ProTaper was taken to length in eight canals. The final diameter of the apical preparation was $40 / 100 \mathrm{~mm}$. The canals were filled with a medium size $\mathrm{g}-\mathrm{p}$ cone coated with AH plus and filled the same way as in group 1.

- Group 4 (Resilon/Real Seal, apical preparation size 40/ $100 \mathrm{~mm}$ ): Eight root canals prepared to size F4 were filled with a medium size Resilon master cone same way as group 2 .
- Group 5 (Gutta-percha/AHplus, apical preparation size $60 / 100 \mathrm{~mm}$ ): Eight root canals were prepared to size 60 with F5 followed by a push pull movement with K 55 and 60 files until file K 60 reached WL. A medium size gutta-percha cone with AH plus was used to fill the canal in the same way as group 1.

- Group 6 (Resilon/Real Seal, apical preparation size 60/ $100 \mathrm{~mm}$ ): Eight root canals were prepared to size 60 with F5 ProTaper followed by a push pull movement with K55 and 60 files until file K60 reached working length. A medium size Resilon/Real Seal was used to fill the canals the same way as group 2 .

All roots sealed with Resilon/Real Seal were light-cured for 40 seconds from the coronal aspect.

\section{Sectioning and Image Analysis}

For each specimen horizontal sections were obtained every 1 up to $3 \mathrm{~mm}$ from the apical foramen. Each section was then digitally photographed using a stereomicroscope. The images were transferred to a computer and a software program (Visilog 5.3 C Noesis 2000) was used to measure the surface area obturated with each technique and to calculate the surface area that was devoid of obturating material (Fig. 1). The surface areas devoid of material (voids) present at each level, for each apical diameter and for each obturation material were statistically compared (Fig. 3).

\section{Scanning Electron Microscope (SEM) Observation}

The sections were mounted onto aluminum stubs using an adhesive carbon tab and placed into a dry heat oven at $50^{\circ} \mathrm{C}$ for 48 hours for complete dehydration. The sections were then sputter coated with a thin gold coating prior to observation using a SEM (Figs 2 and 4).

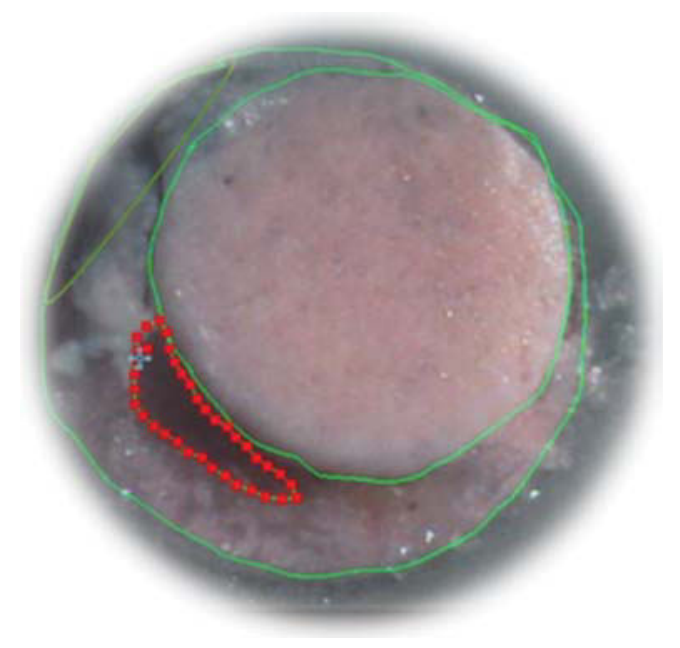

Fig. 1: Measuring the void areas using a software program 

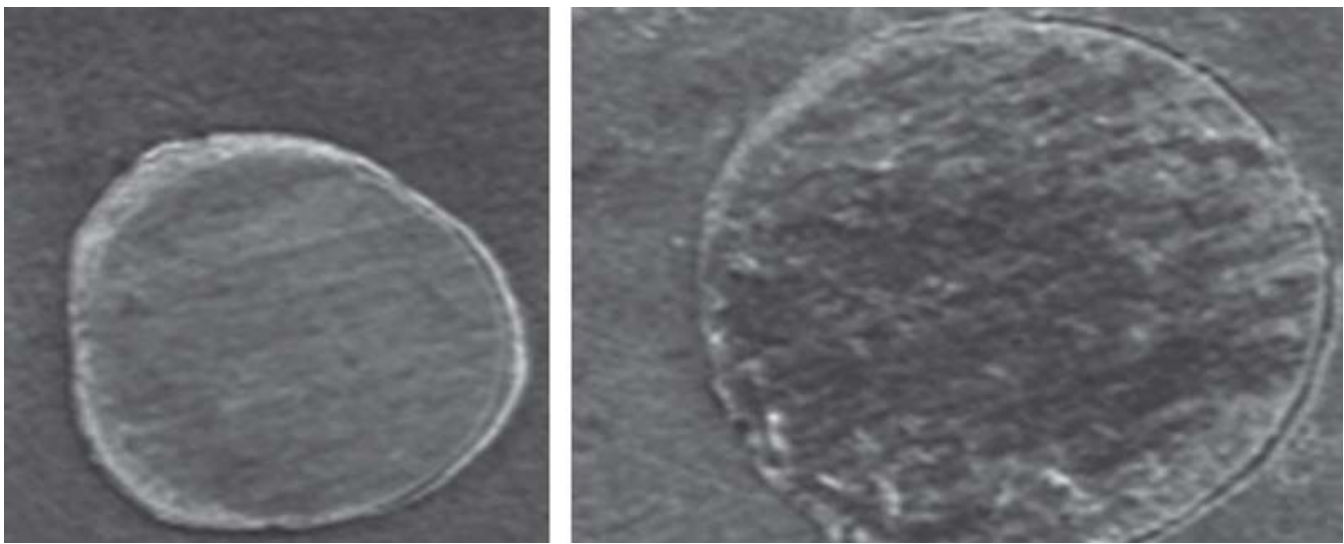

Fig. 2: SEM observations for gutta-percha sample (on the left) and for Resilon/Real Seal sample (on the right) $\times 200$ magnification
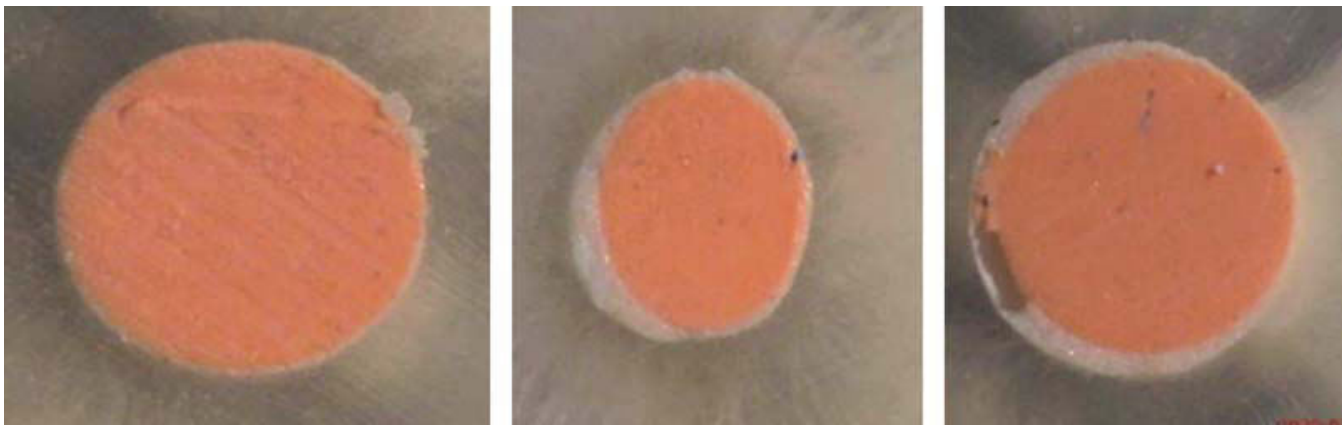

Fig. 3: Three sections of gutta-percha sample $0.25 \mathrm{~mm}$ at 1,2 and $3 \mathrm{~mm}$ from apex
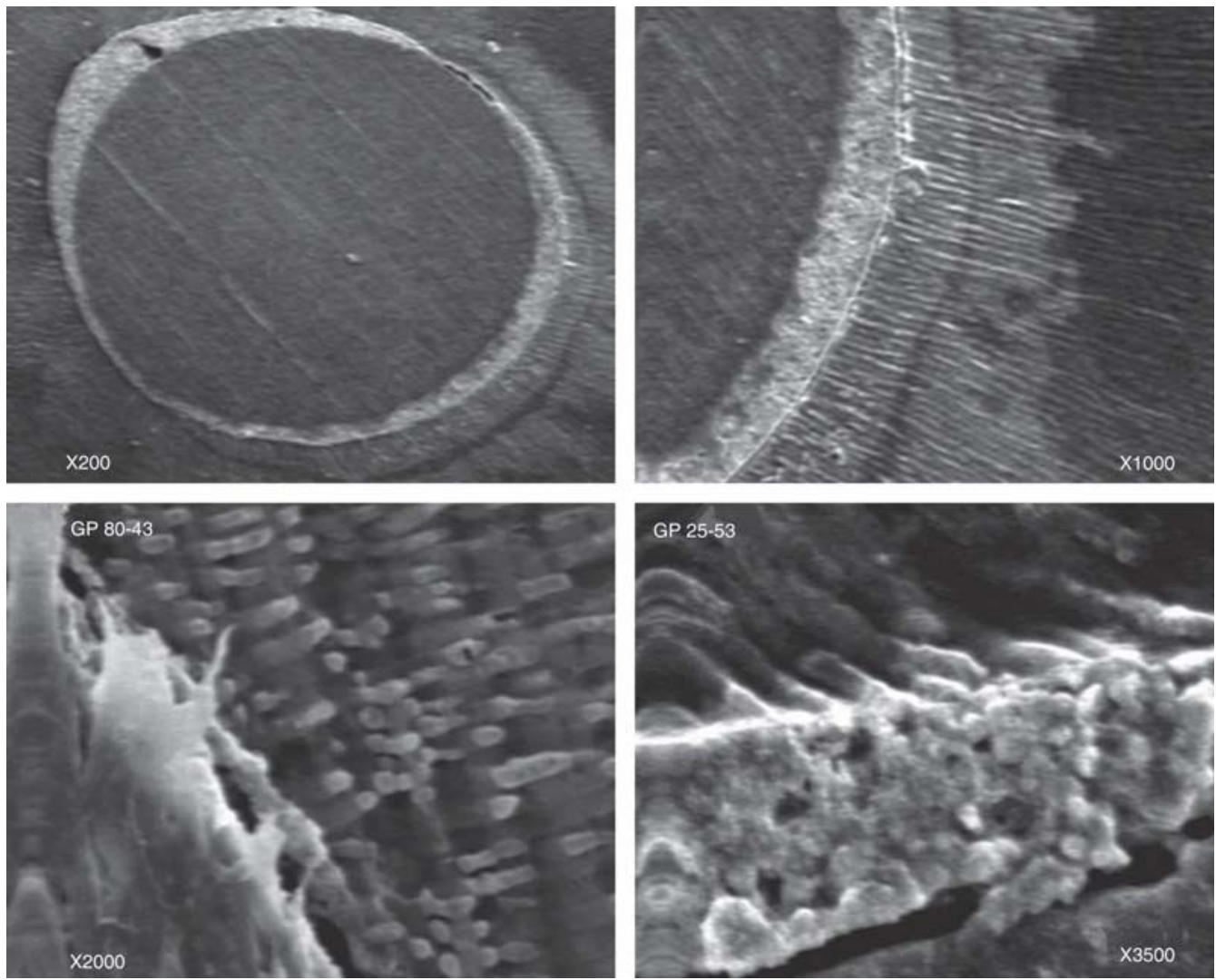

Fig. 4: SEM for gutta percha/AH plus sample respectively $(\times 200, \times 1000, \times 2000, \times 3500)$ 


\section{Data Analysis}

The surface area devoid of material was calculated and statistically analyzed with a statistical software program STATVIEW 2000. For each section (1-3 mm), statistical comparisons between the gutta-percha/AH plus and the Resilon/Real Seal groups were made with Mann-Whitney U-test with Sidak correction. Differences within each obturation group (gutta-percha and Resilon), for each level of sectioning, were analyzed statistically with KruskalWallis test. Significance was set at the $5 \%$ level $(p>0.05)$.

\section{RESULTS}

At 1,2 and $3 \mathrm{~mm}$ level and when comparing the three different apical preparations $(25,40$ and 60$)$ of the groups gutta-percha/AH plus, there were no statistical significant difference in terms of means of voids. The results were the same for all sections when comparing Resilon/Real Seal to the three apical preparations. When comparing the mean of voids between the gutta-percha/AH plus and Resilon/Real Seal at the apical diameter of 25 and 40, there was no statistical difference for all three sections. However, when comparing the two groups with an apical diameter of 60 , the difference was statistically significant.

Resilon/Real Seal group in all three sections had significantly more voids and especially at the $3 \mathrm{~mm}$ level than the gutta-percha/AH plus group (Fig. 5).

\section{DISCUSSION AND CONCLUSION}

Within the experimental conditions of this study, both obturation systems displayed similar amounts of the sealer and void component, regardless of the levels of sectioning investigated and the apical enlargement. The only significant difference was obtained for the apical enlargement to 60 between Real Seal and AH plus sealer.

Because, coronal microleakage $\mathrm{e}^{3,5}$ has also been cited as a significant cause of posttreatment endodontic failure, further studies should be conducted to assess the adaptation of the tested obturation systems in the coronal third.

\section{REFERENCES}

1. Onay EO, Ungor M, Orucoglu H. An in vitro evaluation of the apical sealing ability of a new resin-based root canal obturation system. J Endod 2006;32:976-78.

2. Wu MK, De Groot SD, Van der Sluis LWM, Wesselink PR. The effect of using an inverted master cone in a lateral compaction technique on the density of the gutta-percha fill. Oral Surg Oral Medi Oral Path Oral Radio and Endodontics 2003;96:345-50.

3. Weiss MV, Parashos P, Messer HH. Effect of obturation technique on sealer cement thickness and dentinal tubule penetration. Int Endod J 2004;37:653-63.

4. Epley SR, Fleishman J, Hartwell G, Cicalese C. Completeness of root canal obturations: Epiphany techniques versus guttapercha techniques. J Endod 2006;32:541-44.

5. Gulsahi K, Cehreli ZC, Onay EO, Tasman-Dagli F, Ungor M. Comparison of the area of resin-based sealer and voids in roots obturated with resilon and gutta-percha. J Endod 2007;33: 1338-41.

6. Wu MK, Van der Sluis LWM, Wesselink PR. A preliminary study of the percentage of gutta-percha-filled area in the apical
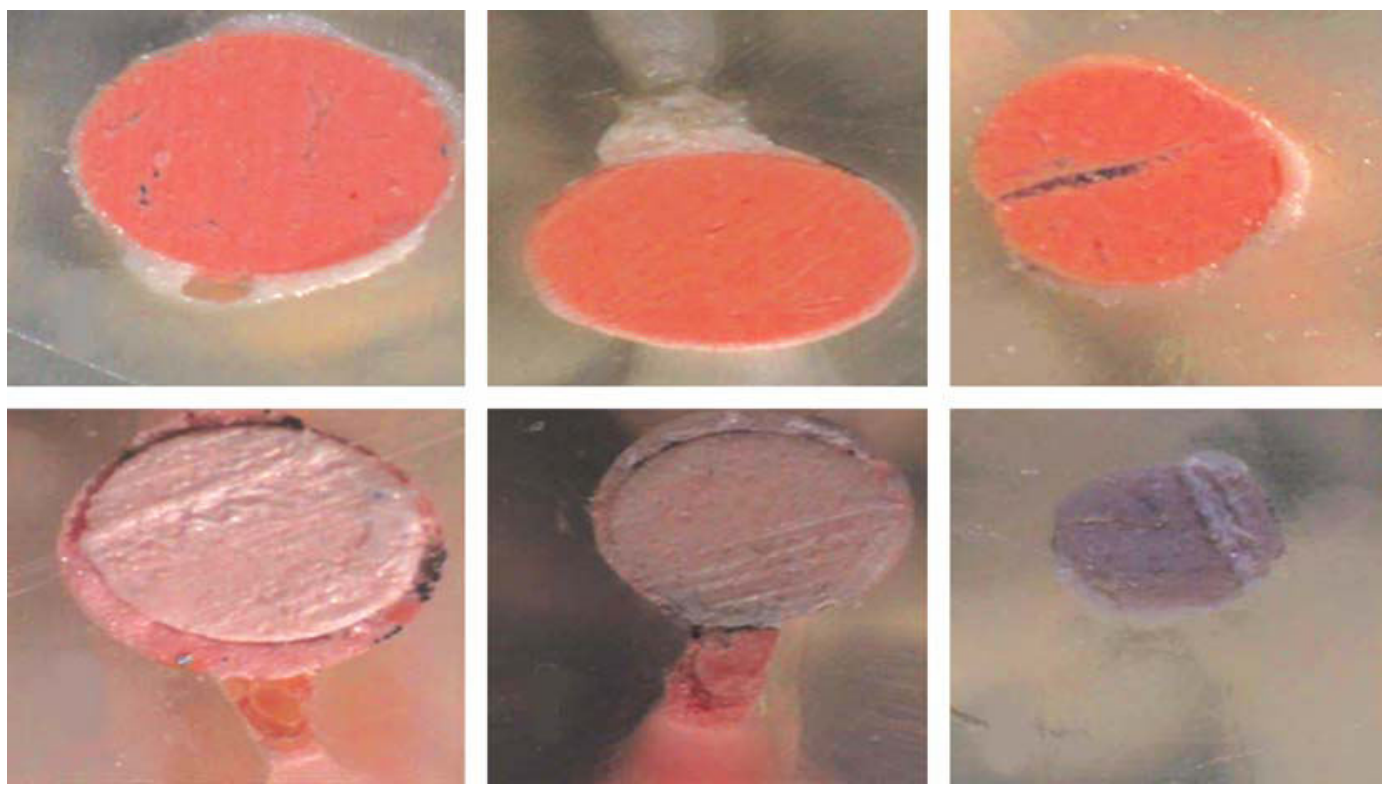

Fig. 5: Sections of gutta-percha sample 0.60 (up) and Resilon sample (down) at 1, 2 and $3 \mathrm{~mm}$ 
canal filled with vertically compacted warm gutta-percha. Int Endod J 2000;35:527-35.

\section{ABOUT THE AUTHORS}

\section{Carla Zogheib (Corresponding Author)}

Senior Lecturer, Department of Endodontics, Faculty of Dentistry St. Joseph University, Beirut, Lebanon, Phone: 009613304694 e-mail:zogheibcarla@gmail.com

\section{Alfred Naaman}

Head, Department of Endodontics, Faculty of Dentistry, St. Joseph University, Beirut, Lebanon

\section{Etienne Medioni}

Vice Dean for International Relations, Department of Conservative Dentistry and Endodontics, Faculty of Dentistry, Nice-Sophia Antipolis, Nice, France 